\title{
Phenotype and cell proliferation activity of duct- like structures in human sublingual glands: histological and immunohistochemical study
}

\author{
Elen de Souza TOLENTINO1', Cleverson Soares TEIXEIRA², Luciana Reis AZEVEDO-ALANIS ${ }^{3}$, Heitor Marques \\ HONÓRIO ${ }^{4}$, José Humberto DAMANTE ${ }^{5}$ \\ 1- Department of Dentistry, Maringá State University, Maringá, PR, Brazil. \\ 2- Lauro de Souza Lima Institute, Bauru, SP, Brazil. \\ 3- Department of Dentistry, Pontifical Catholic University of Paraná, Curitiba, PR, Brazil. \\ 4- Department of Pediatric Dentistry, Orthodontics and Community Health, Bauru School of Dentistry, University of São Paulo, Bauru, SP, Brazil. \\ 5- Department of Stomatology, Bauru School of Dentistry, University of São Paulo, Bauru, SP, Brazil.
}

Corresponding address: Elen de Souza Tolentino - Av. Mandacaru, 1550 -Bloco S08 - 87083-170 - Maringá - PR - Brazil - Phone: +55 (44) $2101-9051$ e-mail: elen_tolentinon@hotmail.com

Submitted: September 1, 2014 - Modification: October 30, 2014 - Accepted: November 5, 2014

\section{ABSTRACT}

\begin{abstract}
There are several age-related microscopic changes in the salivary glands, including the increase in the number of duct-like structures (DLS). However, the true origin and the phenotype of the DLS are not known. Objective:To evaluate the phenotype and the cell proliferation index of the DLS of human sublingual glands. Material and Methods: Sixty sublingual glands obtained from human cadavers were divided into two groups - 0-30 and 61-90 years old. The phenotype was estimated by immunostaining for cytokeratin 19 (CK 19) and the S-100 protein as well as by the presence of mucin and glycogen. The cell proliferation index was determined by the Ki-67 antibody. The histochemical techniques used periodic acid-Schiff (PAS) and Alcian Blue. In each captured microscopic field, the DLS were counted to establish a percentage for the staining profile. The statistical analysis was accomplished using Student's t-test, the Mann-Whitney test and Pearson's correlation coefficient $(p<0.05)$. Results: Comparing both groups, only CK 19 showed a statistically significant difference $(p=0.033)$, with the strongest expression in the elderly group. There was no significant difference between PAS and Alcian Blue $(p=0.270)$. In both groups, the immunostaining for CK 19 was stronger than that for $S-100(p=0.004 ; p<0.001)$, but there was no correlation between the two immunomarkers $(\rho=-0.163 ; p=0.315)$. There was no immunostaining for Ki-67. Conclusions: DLS demonstrate a ductal phenotypic profile and do not present cell proliferation activity. DLS may represent a regressive process arising from acini or represent the result of metaplasia.
\end{abstract}

Keywords: Sublingual gland. Salivary ducts. Aging. Histology. Immunohistochemistry

\section{INTRODUCTION}

Age-related changes in human salivary glands have been described by some studies in humans ${ }^{2,6-9,18,23,25,29,30}$ and animals ${ }^{20,24}$. The literature regarding aging alterations in human sublingual glands is limited by the number of reports, and some phenomena are still unclear. Despite the different methodologies used in the investigations of age-related microscopic changes in the salivary glands, the consensus is that there is a decrease in the volume of parenchyma that is accompanied by an increase in fat and connective tissue $e^{2,6,7,9,18,23,28-30}$.

This aging process also includes acinar atrophy and an increase in the number of duct-like structures (DLS) 2,6,8,9,14,18,23,24 (Figure 1). The term "duct-like structures" is commonly used because there is no evidence that the structures are true ducts. For many researchers, the appearance of DLS with age represents the final process of acinar atrophy, and the making the distinction between a duct and a DLS can be very difficult ${ }^{2,5,6,9,18,24,25,28}$. 
Furthermore, the DLS do not represent a specific phenomenon of senescence, and they also appear in atrophic glands $\mathbf{s}^{5,28}$ and glandular neoplasms $\mathbf{s}^{1,12}$.

Some authors also believe that, during the aging process, atrophic acini can resemble ducts, and the distinguishing between them can be very difficult or even impossible $2,5-7,9,18,22,23,28$, demonstrating that the morphology of aged salivary glands is not completely established.

Molecular alterations of the sublingual glands during the aging process are not known. The origin and cellular constitution of DLS is not elucidated yet, for example. Regarding immunohistochemical studies related to the salivary glands, most of them only aim to characterize their epithelial and/ or myoepithelial components $3,5,10,13,28$ or to study pathological processes such as neoplasias ${ }^{1,12}$. It is evident that the literature regarding age-related changes in salivary glands is not abundant. In humans, studies are scarce and the sublingual gland is rarely contemplated.

The increase in the number of DLS allows speculation that a mitotic process is occurring within the cells or even a transition of an acinus to a duct ${ }^{15,28}$ because the acini disappear while the DLS increase in number. The true origin and the phenotype of the DLS are not known. Are they atrophic acini or true ducts? The aim of this work is to evaluate the phenotype and the cell proliferation index of DLS in human sublingual glands during the aging process.

\section{MATERIAL AND METHODS}

This study was approved by the Human Research Ethics Committee of the Bauru School of Dentistry at the University of São Paulo (USP) (Process $n^{\circ}$ 59/2010) and also by the São Paulo Death Verification Service (USP), where the glands were obtained. All experiments followed the guidelines of the Helsinki Declaration.

\section{Samples}

Sixty human sublingual glands were obtained from human cadavers during autopsies. The methods and the exclusion criteria were the same as those used by Azevedo, et al. ${ }^{2}$ (2005). Individuals with the following systemic disorders or base diseases were excluded from the study: Iymphoma, leukemia, mucoviscidosis, rheumatic diseases, Sjögren's syndrome, obesity, cachexia, diabetes mellitus, alcoholic cirrhosis, collagen diseases, history of head or neck cancer-related surgery, history of cytotoxic drug administration, previous radiotherapy of the head or neck during the last 3 months and macroscopic autolysis. Data regarding systemic involvement and cause of death were obtained from the family and the autopsy reports.
The main causes of death included pulmonary edema, bronchopneumonia, acute myocardial infarction, cerebral vascular accident, ischemic heart disease, cerebral edema and congestive heart failure. The interval between the time of death and the autopsy ranged from 6:05 to $92: 55 \mathrm{~h}$, with a mean time of 16:46 h. The cadaver glands were completely removed along with a portion of the overlying mucosa of the mouth floor.

The glands from the cadavers were divided into two groups: group I (control; 0-30 years old) and group II (60-90 years old). The specimens were fixed in $10 \%$ formalin and processed using routine procedures. The paraffin-embedded material was cut into 3-5 $\mu \mathrm{m}$-thick sequential slices.

\section{Phenotype study}

For the study of the cellular phenotype of the DLS, antibodies against cytokeratin 19 (CK 19; clone RCK108; isotype IgG1/mouse; monoclonal; DAKO, Dako Inc, Carpinteria, CA, USA, cod\#M0888; dilution 1:100) and the S-100 protein (S-100; isotype IgG/rabbit; polyclonal; DAKO, Dako Inc, Carpinteria, CA, USA, cod\#Z0311; dilution 1:2000) were used. The anti-CK 19 antibody was used to stain the cells of the ductal system ${ }^{3,10,13,16}$ and anti $\mathrm{S}-100$ to stain the serous acinar cells ${ }^{19,21}$. The histochemical markers periodic acid-Schiff (PAS) and Alcian Blue ( $\mathrm{pH} 2.5)$ were used to stain the mucous acinar cells. For the analysis of the cell proliferation capacity of the DLS, the Ki-67 (Ki-67; clone MIB-1; isotype IgG1/mouse; monoclonal; DAKO, Dako Inc, Carpinteria, CA, USA, cod\#M720; dilution $1: 400$ ) antibody was used ${ }^{4,11,13}$.

The immunohistochemical study was performed using the polymeric horseradish peroxidase (HRP)linker antibody conjugate technique. The material was incubated in a humidity chamber at $4^{\circ} \mathrm{C}$ for 18 hours. Considering the large S-100 protein family, the polyclonal rabbit anti-S-100 was used, which reacts strongly with all family members, including human S100A and B sub-units. The specimens were counterstained with Harris hematoxylin, and the slides were mounted using adhesive resin (Entellan ${ }^{\circledR}$, Merck, Darmstadt, Hessen, Germany) and coverslips. For all immunohistochemical markers, both a positive and a negative control were prepared according to the manufacturers recommendations.

The same examiner analyzed all slides. For analysis, a digital image-capture computer system (Axiocam MRc, ZEISS, Jena, Thuringia, Germany) and a binocular microscope (Axioskop 2 Plus, ZEISS, Jena, Thuringia, Germany) with a $40 \mathrm{X}$ objective were used. The images were analyzed using the software supplied with the microscope (Axiovision 4.5, ZEISS, Jena, Thuringia, Germany). Each microscopic field was previously 
standardized in this program to avoid differences in the expression intensity of the antibodies. In each captured microscopic field, the DLS were counted to establish their percentage in the staining profile.

The most representative areas of the event (spots), identified using a lower magnification, were selected. The spots comprised the areas of the highest concentration of structures. When the DLS were diffusely distributed and spread out, 20 spots were randomly selected. If the number of spots was less than 20, all fields were analyzed. In group I, all microscopic fields containing DLS were captured and in group II, 20 fields containing DLS were randomly captured (Figure 2). True ducts or acini that were easily recognized were excluded from the count.

For the localization and the counting of the DLS, slides stained with hematoxylin-eosin (H.E.) were used. We considered DLS to be those with shrunken seromucous cells, granule depletion and widened lumens that were adjacent to ductal epithelial cells and resembled intralobular non-striated ducts, which is consistent with the characterization used in our previous study ${ }^{9}$. We excluded true excretory ducts, which exhibited a dilated lumen lined by cuboidal cells with central nuclei and an eosinophilic cytoplasm 22.

A section of each gland was analyzed with each studied molecule, totaling 60 slides for each staining (30 per group). Therefore, a total of 360 slides were analyzed [60 for each marker: H.E., PAS, Alcian Blue ( $\mathrm{pH} 2.5)$, CK 19, S-100 and Ki-67].

A semi-quantitative scoring system was applied to the immunohistochemical and histochemical analysis [adapted from Soini, et al. $\left.{ }^{26}(2001)\right]$. Both analyses resulted in a final score that represented no expression, weak expression or strong expression. In each microscopic field, the percentage of positive (with partial or total staining) DLS was calculated for each marker. Scores ranging from 0 to 4 were
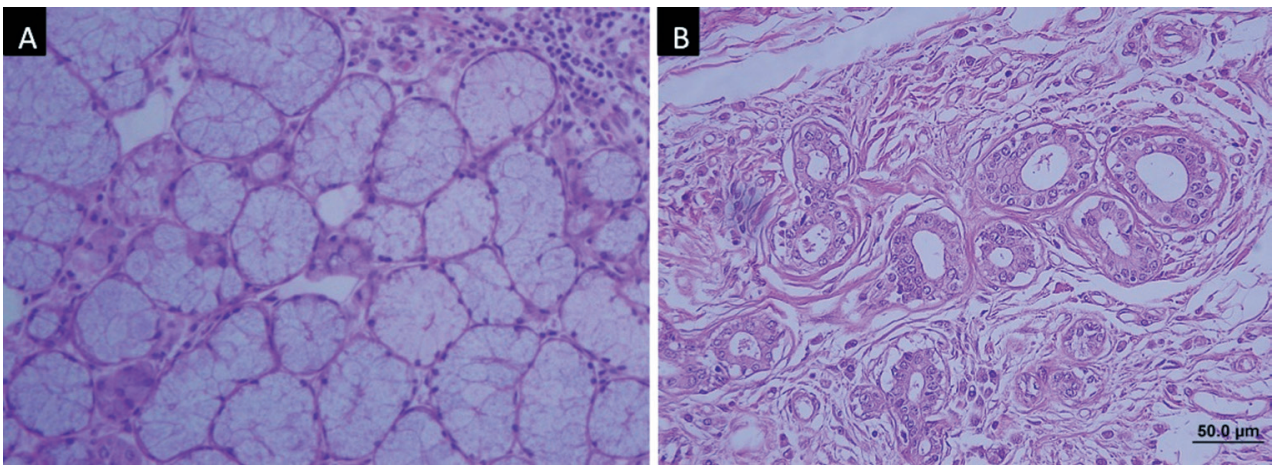

Figure 1- A: Normal gland - 26 years old (Group I). Preserved acini and absence of duct-like structures (DLS) are observed; B: Altered gland - Presence of DLS, absence of acini and substitution of the glandular parenchyma by connective tissue 68 years old (Group II) (hematoxylin and eosin 100X)

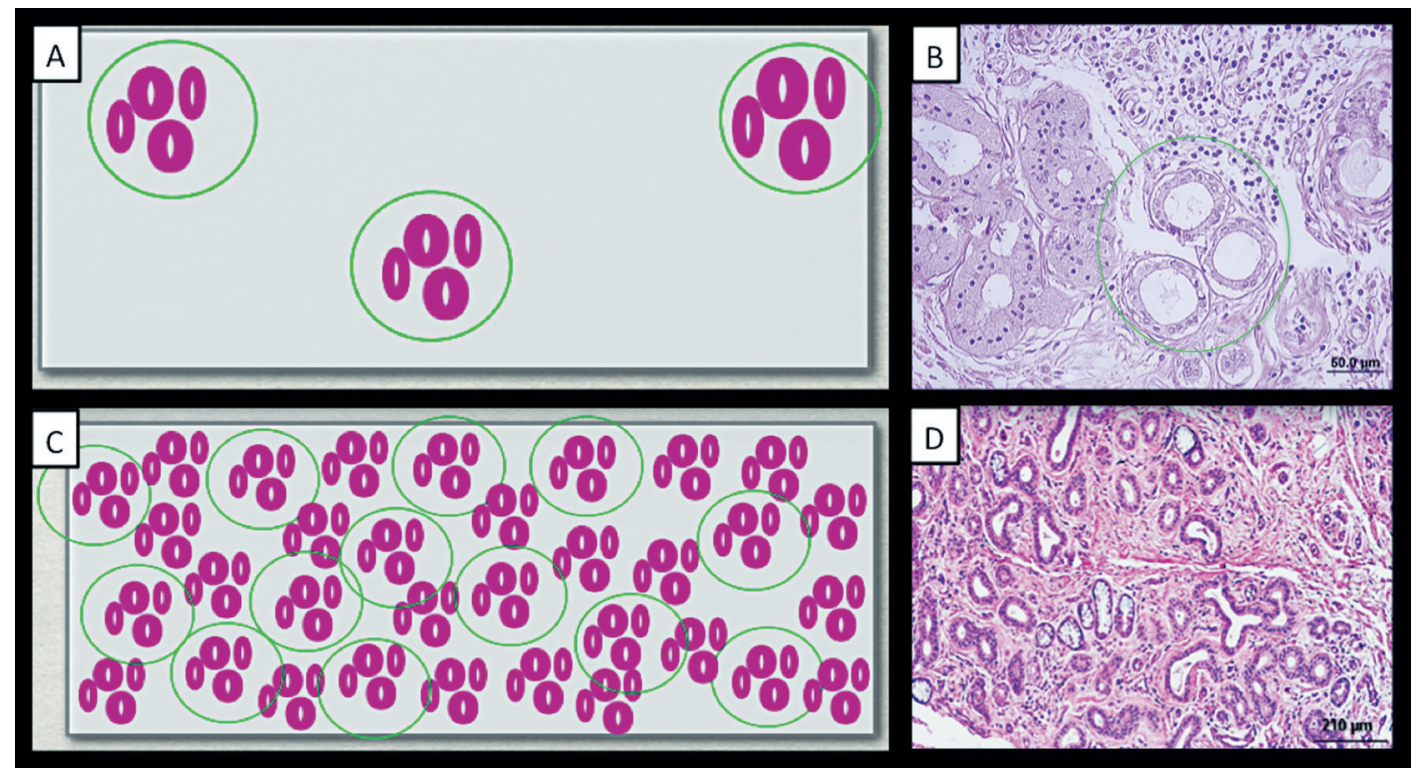

Figure 2- Schematic representation of the selection of spots. A: Due to the small quantity of duct-like structures (DLS) in group I, all microscopic fields containing DLS were captured; B: A spot containing three DLS; C: 20 fields containing DLS were randomly captured in group II; D: Diffuse distribution of DLS in group II 
attributed to those percentage values: 0) absence of positive DLS; 1 ) $<25 \%$ of positive DLS; 2 ) $26-50 \%$ of positive DLS; 3) $51-75 \%$ of positive DLS; 4) $>75 \%$ of positive DLS. Then, for the DLS positive samples, the partial or total covering and the intensity of the immunostaining in the positive cells were calculated following the same method applied previously. Finally, the sum of the values attributed to the quantity, covering and intensity of the immunostaining resulted in a final score where 3-7 represented weak to moderate expression and 8-12 was a strong expression of the antibody.

Basically, the PAS and Alcian Blue staining in the DLS followed the same scoring system, except for the intensity values, which did not exist for these stains.

Table 1- Comparison between groups regarding histochemical and immunohistochemical staining

\begin{tabular}{|c|c|c|c|c|c|c|c|}
\hline \multicolumn{8}{|c|}{ Groups } \\
\hline & & $I$ & & & II & & $p$ \\
\hline & & $(n=12)$ & & & $(n=28)$ & & \\
\hline & Median & $1^{\text {st }}$ Quartile & $3^{\text {rd }}$ Quartile & Median & $1^{\text {st }}$ Quartile & $3^{\text {rd }}$ Quartile & \\
\hline S-100 & 0.000 & 0.000 & 6.000 & 1.286 & 0.000 & 3.875 & 1.000 \\
\hline CK-19 & 9.000 & 6.000 & 11.625 & 10.840 & 9.083 & 12.000 & $0.033^{*}$ \\
\hline PAS & 0.000 & 0.000 & 0.000 & 0.000 & 0.000 & 0.458 & 0.536 \\
\hline AA & 0.000 & 0.000 & 1.875 & 0.000 & 0.000 & 0.917 & 0.907 \\
\hline
\end{tabular}

Mann-Whitney test $(p<0.05)$. *Statistically significant. AB - Alcian Blue

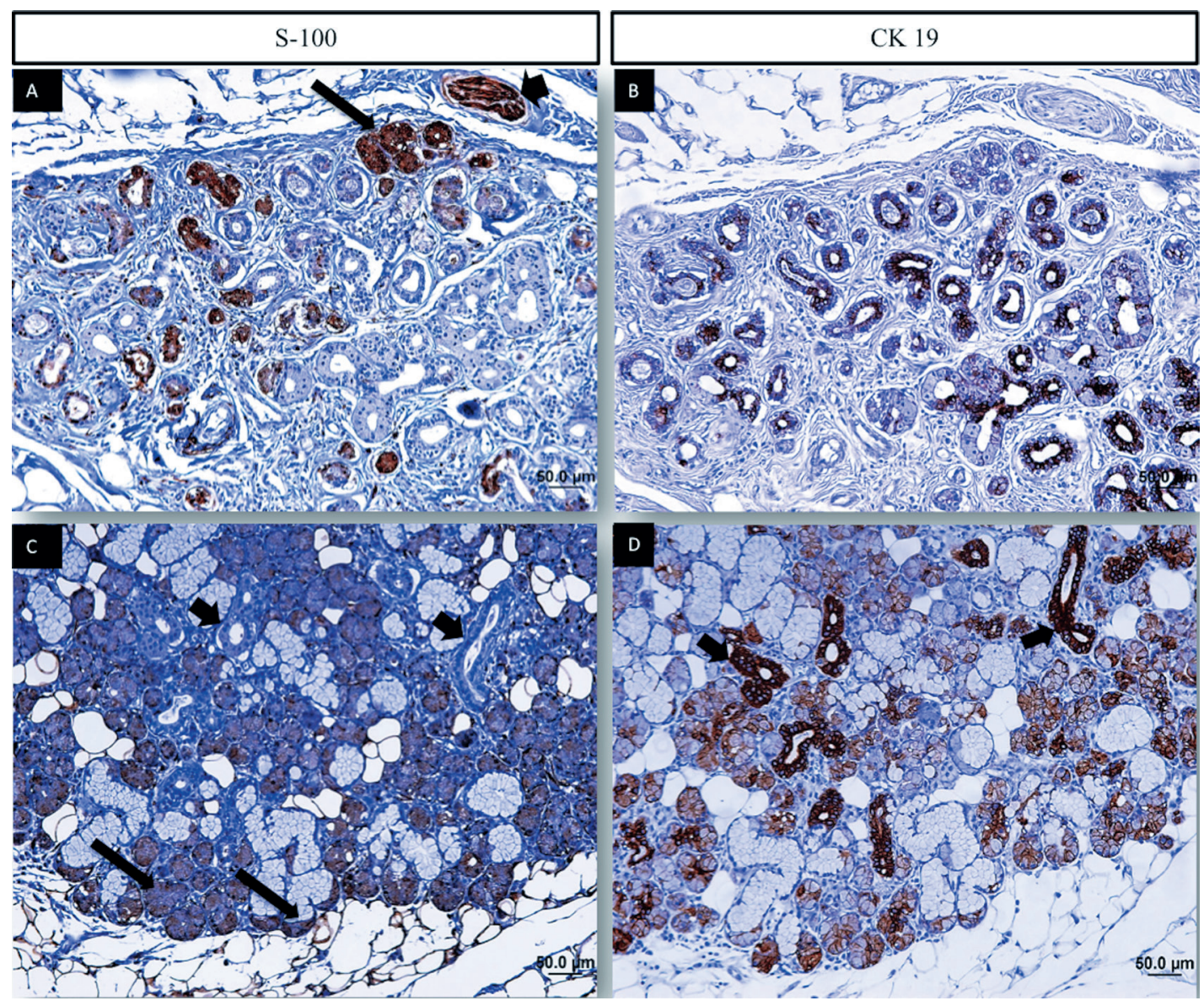

Figure 3- A: Duct-like structures (DLS) with weak or absent S-100 expression. A strong expression of S100 seen in the nerve cells (short arrow) can be regarded as the positive control. Serous cells with strong expression (arrow) (Group II); B: Strong expression of CK 19 in some cells in the DLS. Serous cells with weak expression (Group II); C: Positive S-100 expression in serous acinar cells (arrows) and negative in ducts and DLS (short arrows) (Group I); D: Strong CK 19 expression in ducts and DLS (arrows) and weak or moderate expression in serous acinar cells (Group II) 


\section{Cellular proliferative activity study}

The cellular proliferative activity in the DLS, revealed by Ki-67 staining, was calculated as the percentage of positive cells to establish a cellular proliferation index in the same previously captured fields.

\section{Statistical analysis}

The comparison between groups and between markers was accomplished by the Student's t-test or the Mann-Whitney test. The correlation among the staining groups was established by the Spearman correlation coefficient. The level of significance was set at $5 \%$ for all tests. A Kappa test was used to evaluate intraexaminer concordance. Thirty slides were randomly re-evaluated 2 months after the initial analysis.

\section{RESULTS}

The Kappa coefficients (K) for the S-100, CK 19, PAS and Alcian Blue variables were $87.50 \%$, $78.13 \%, 96.88 \%$, and $98.88 \%$, respectively.
Phenotype study

The sublingual glands of both groups showed great variability in their histological architecture. This variation could be observed among different glands or even among different lobes of the same gland. Overall, the glands of young people were intact, with serous and mucous acini well delineated and absent or few and sparse DLS (Figure 1A). In the glands of the elderly, certain sections had preserved lobes while others had characteristics inherent to senility, which is indicated by the intense replacement of the glandular parenchyma by fibrous and fatty tissue and many DLS (Figure 1B). In group I, from the 30 slides analyzed, only 12 had DLS, whereas in group II, only 2 slides did not show these structures.

When comparing both groups, only the immunostaining for CK 19 showed a statistically significant difference between them $(p=0.033)$, with stronger expression in the elderly group. Although the expression for $\mathrm{S}-100$ was also stronger in group II, the difference was not significant $(p=1.000)$ (Table 1).

Table 2- Comparison between S-100 and CK 19 immunostaining of duct-like structures in groups I and II

\begin{tabular}{|c|c|c|c|c|c|c|}
\hline \multicolumn{7}{|c|}{ Group Ia } \\
\hline & $s-100$ & & & ck 19 & & \\
\hline Mean & SD & SEM & Mean & SD & SEM & $p$ \\
\hline 2.583 & 3.370 & 0.973 & 7.625 & 4.216 & 1.217 & $0.004^{*}$ \\
\hline \multicolumn{7}{|c|}{ Group II $^{b}$} \\
\hline & $S-100$ & & & CK 19 & & \\
\hline Median & $1^{\text {st }}$ quartile & $3^{\text {rd }}$ quartile & Median & $1^{\text {st }}$ quartile & $3^{\text {rd }}$ quartile & \\
\hline 1.286 & 0.000 & 3.875 & 10.840 & 9.083 & 12.000 & $<0.001^{*}$ \\
\hline
\end{tabular}

aStudent's t-test $(p<0.05)$. SD - standard deviation. SEM - standard error of mean. Group I (0-30 years old). ${ }^{\mathrm{b}}$ Mann-Whitney test $(\mathrm{p}<0.05)$. Group II (61-90 years).
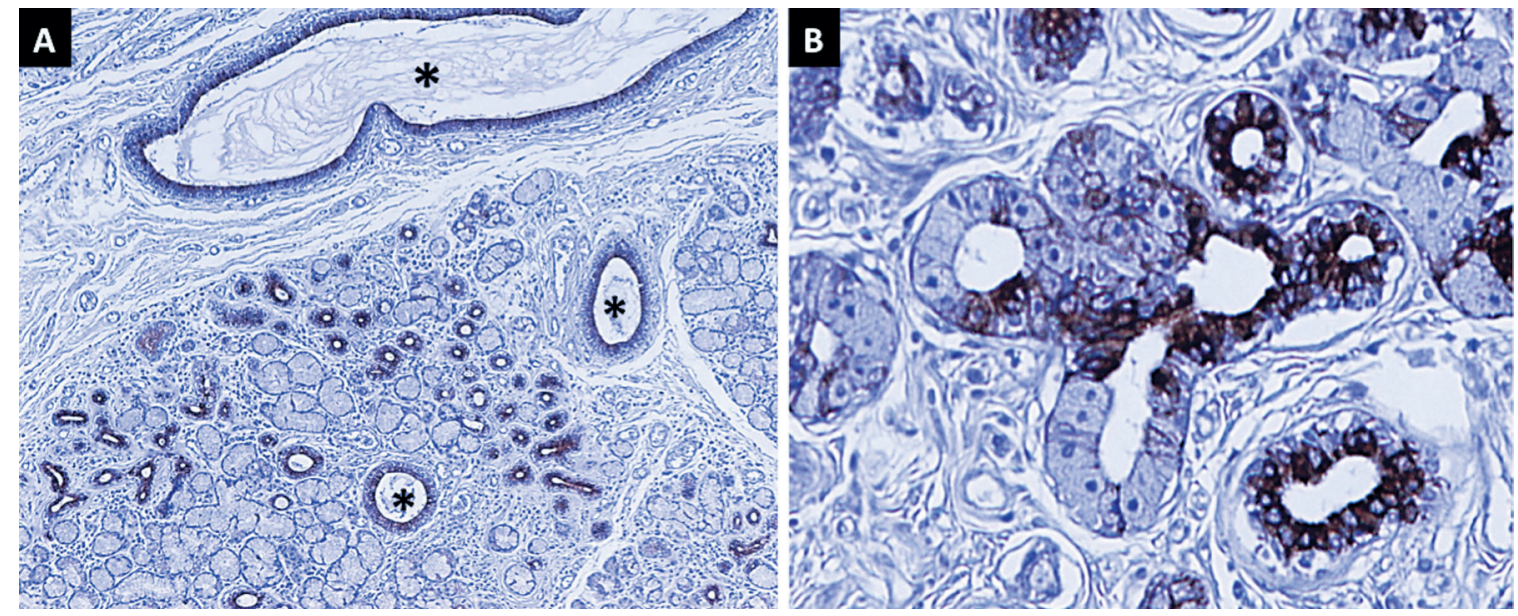

Figure 4- A: Similar CK 19 expression between ducts $\left(^{*}\right)$ and duct-like structures (Group II). B: Mixed structure with ductal and acinar cells. The ductal cells are stained with the anti- CK 19 (Group II). 


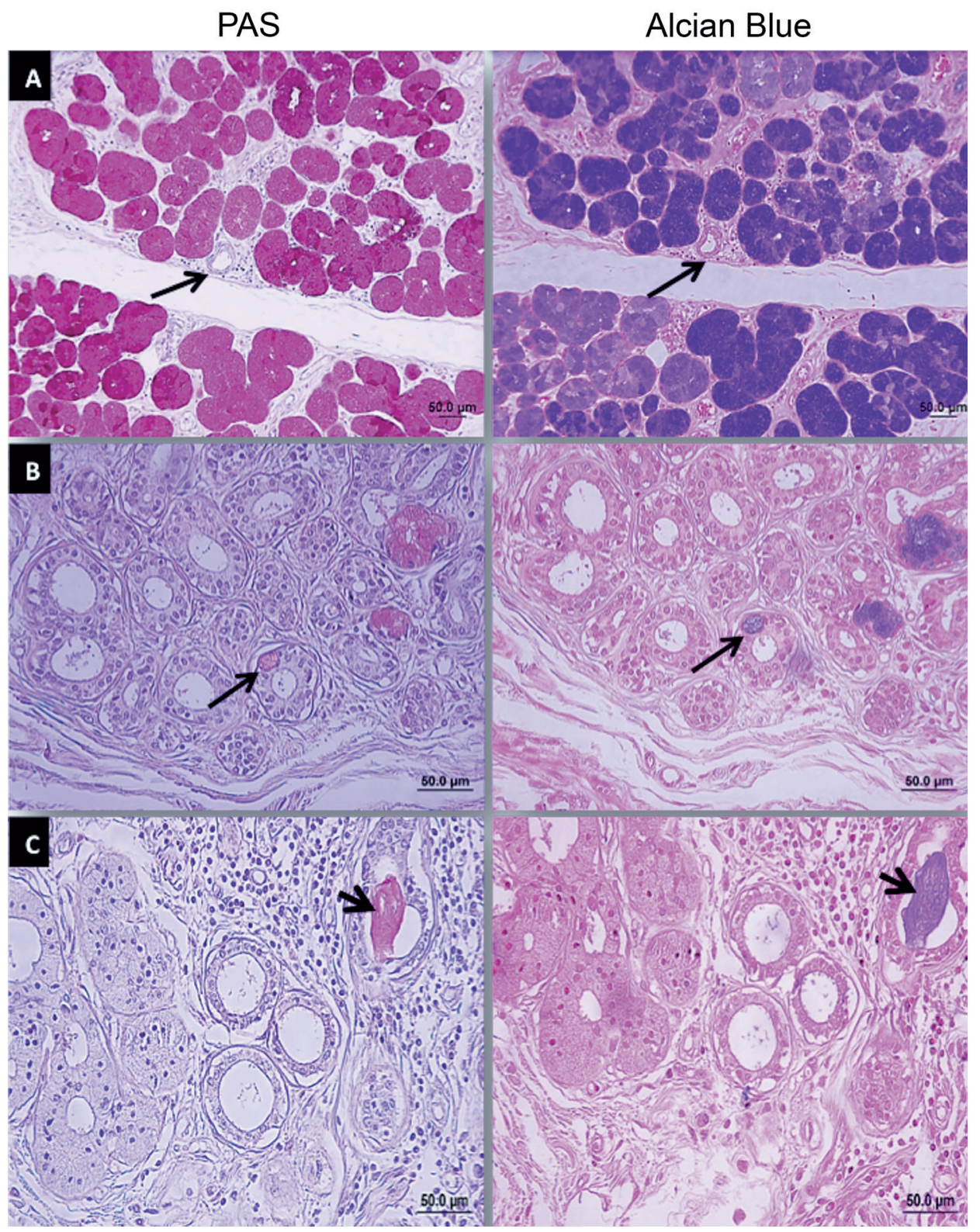

Figure 5- A: Mucous acini stain for periodic acid-Schiff (PAS) and Alcian Blue. The isolated duct-like structures (DLS) is negative for both markers (arrows) - group I (21 years old); B: Partial staining of a DLS for PAS and Alcian Blue (arrows). The most DLS are negative for both markers. Group II (76 years old); C: Negative DLS for PAS and Alcian Blue. Presence of mucus in the lumen of a DLS (arrows). Group II (76 years old)

Serous acini (identified by S-100 expression) were present in the DLS with a lower frequency and a weaker intensity than the CK 19 expression levels. Frequently, the immunostaining for S-100 was absent in the DLS. In contrast, the expression of CK 19 was strong in the DLS and very similar to the immunostaining in the ducts. The serous acini exhibited strong immunostaining for S-100 and moderate, weak or absent expression of CK 19. The ducts were invariably negative for S-100 and positive for CK 19 (Figure 3).

Regarding group I, the immunostaining of the DLS for CK 19 was stronger than for S-100, with a statistically significant difference observed $(p=0.004)$ (Table 2).

For group II, the same circumstance was observed and this difference was even more significant $(p<0.001)$ (Table 2$)$. This condition was also seen in the descriptive microscopic analysis because in the senile glands, the DLS strongly expressed CK 19 (Figure 4A), showing a ductal pattern. In some sections, we also observed the presence of areas with a mixture of acinar and ductal cells (Figure 4B).

When the Pearson correlation coefficient was applied, the correlation between CK 19 and S-100 was absent $(\rho=-0.163 ; p=0.315)$.

Regarding the PAS and Alcian Blue staining for 


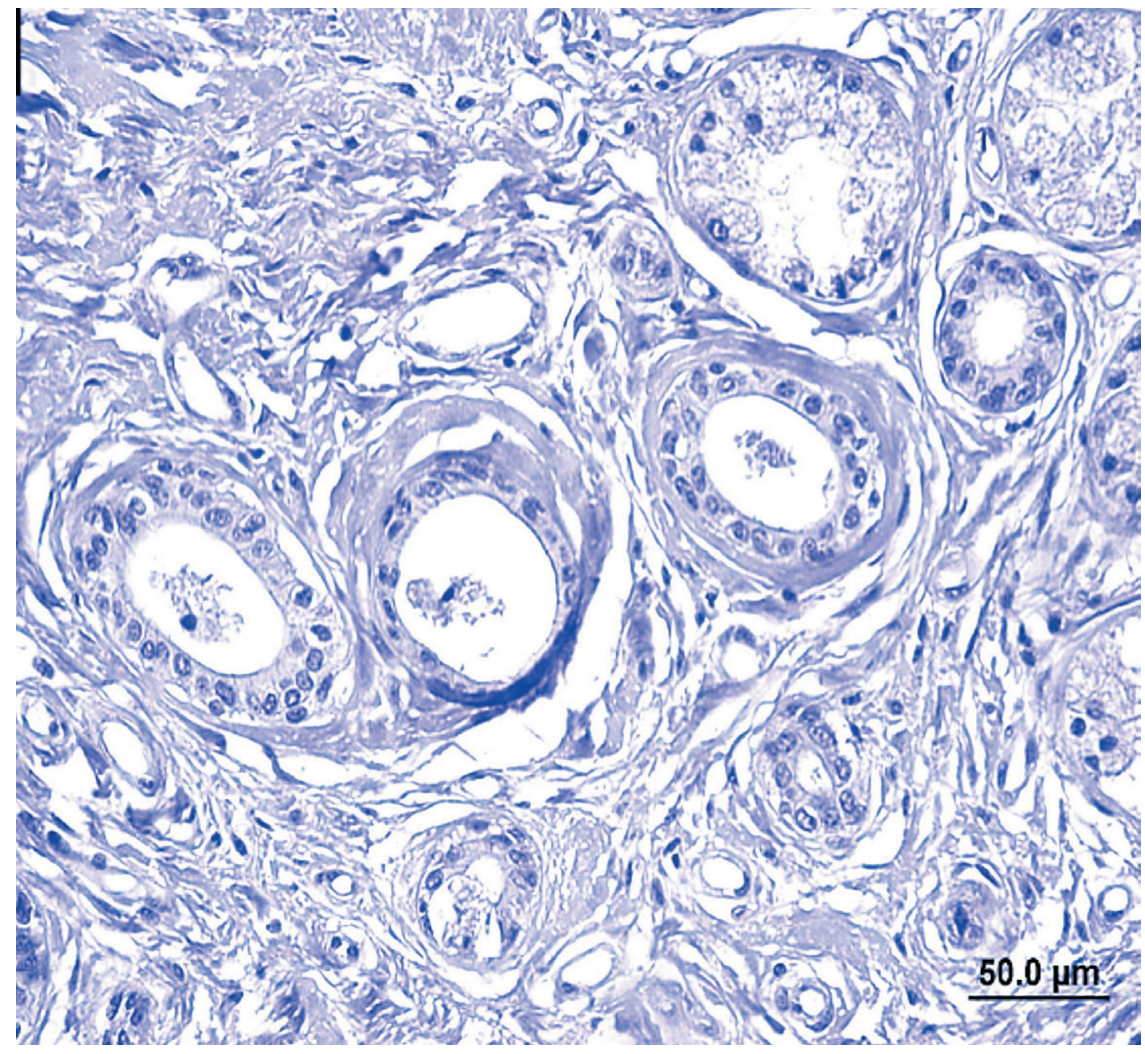

Figure 6- Absence of nuclei expression of Ki-67 in the duct-like structures (Group II)

mucous acini, both markers worked similarly, where the staining was evident in the mucous acini and quite rare in the DLS (Figure 5A). When there was staining, it was restricted to a small portion of the structure and was classified as partial (Figure 5B). These markers also showed, in some cases, the presence of mucin within the ducts or DLS (Figure $5 \mathrm{C})$. There was no statistically significant difference between PAS and Alcian Blue $(p=0.270)$.

\section{Cell proliferation activity study}

In both groups, there was no immunostaining for the Ki-67 antibody in any DLS (Figure 6). The expression of various basal cells of the epithelium of the oral mucosa worked as a positive controlfor the antibody used.

\section{DISCUSSION}

The sublingual glands used in this work are the same as those used by Azevedo, et al. ${ }^{2}$ (2005). In regards to the gender of the cadavers and the side of the glands, previous studies have already demonstrated that there are no differences in these variables ${ }^{2,18,23}$. We consider our samples to be very rich and consistent because the sublingual glands were removed from the cadavers after a short postmortem period. Moreover, the sublingual glands are rarely addressed in previous studies, where they are typically derived from rats ${ }^{20,24,28}$. Only the works of the present line of research use human sublingual glands $2,14,18,22$.

Because DLS are not an exclusive phenomenon of senescence, group I was used in this study as a control to observe whether there were differences in the staining pattern of DLS in this group in comparison to group II. When a gland from group I exhibited DLS, the number was very low and often limited to the extremity of the lobe. We believe that the presence of DLS in the glands of young individuals may be due to some injury, i.e., small traumas to the mouth floor during feeding and microbstructions of the peripheral ductal system. Moreover, these structures have been shown in studies that induce glandular atrophy through the duct obstruction ${ }^{5,28}$ or in pathological processes such as neoplasms ${ }^{1,12}$. Therefore, despite the presence of DLS upon cellular senescence, they are not exclusive in this age group, which justifies why we opted to include group I in the present study.

In regards to normal salivary glands, several studies demonstrate that CK 19 is strongly expressed in all parts of the ductal system and shows weak response to serous and mucous acinar cells $^{3,16}$. For Geiger, et al. ${ }^{10}$ (1987) and Ihrler, et al. ${ }^{13}$ (2002), CK 19 is expressed in ductal cells but completely absent in acinar cells. Regarding the S-100 protein, studies in normal salivary glands and tumors showed strong expression in serous acinar cells and weak expression in the ductal segments ${ }^{19,21}$. In this study, CK 19 was strongly expressed in true ducts and most of the DLS. The 
CK 19 expression in the serous acini was weaker or absent and was not detected in the mucous acini. However, the S-100 protein was expressed in serous acinar cells and absent in true ducts and mucous acinar cells.

The difference in the immunostaining of CK 19 in the DLS between groups was significant $(p=0.033)$ (Table 1). The expression of this protein was higher in the elderly, indicating the possibility that, over time, the DLS begin to express the molecules of the ductal system more strongly.

In both groups, there was a statistically significant difference between CK 19 and S-100 (Tables 4 and 5) with more intense CK 19 staining, demonstrating a ductal pattern of the DLS. It is possible that the DLS increase their ductal pattern with age while they simultaneously decrease the expression of S-100. However, there was no correlation between the immunomarkers ( $\rho=-$ $0.163, p=0.315$ ).

Regarding the cell proliferation activity, we did not find any cells positive for $\mathrm{Ki}-67$ in the DLS of both groups (Figure 6). The effectiveness of the antibody used was proven by the presence of nuclear staining in various basal cells of the oral mucosa epithelium in the same sections. A similar situation was described by Dayan, et al.7 (2002) who found a proliferation index from $0 \%$ in acinar and ductal cells from young and senile human labial glands.

According to Takahashi, et al. ${ }^{28}$ (2002), the staining for PCNA in ductal cells is high in the early stages of glandular atrophy and subsequently decreases. Instead, the DLS are commonly observed in the later stages of atrophy, suggesting that both mitosis (early stages) and the transition from acinar to ductal cells (later stages) contribute to the increase in the number of ducts in the atrophic sublingual glands. These findings may explain the absence of immunostaining for Ki-67 in the DLS, demonstrating that they represent a late stage of atrophy (or aging) where mitosis is no longer observed. However, even in the DLS of young glands, there was no evidence of cell proliferation, which is difficult to explain unless the authors used another marker (PCNA). Based on our results, we do not support the hypothesis that mitosis explains the increase in the number of DLS.

If we consider the suggestions by Scott, et al. ${ }^{25}$ (1987) and Takahashi, et al. ${ }^{28}$ (2002) that the DLS represent the final process of acinar atrophy, we can infer that, in this investigation, S-100 was expressed in the DLS due to the presence of serous acinar cells that remained from the atrophy process. Unlike serous cells, there was rarely any trace of mucous cells in the DLS (Figure 5B). Therefore, we hypothesized that, if these structures are actually products of an acinar regression, this acinus is mixed, where mucous cells degenerate while the serous portion is more resistant and occasionally remains active. This finding corroborates other studies in humans ${ }^{2,18,29}$, in which the serous cells showed greater resistance to autolysis compared to the mucous cells.

Frequently, the areas of mixed serous and ductal cells were visualized as illustrated in Figure 4B. Acini also have been reported to de-differentiate into ducts ${ }^{15}$. Ihrler, et al. ${ }^{13}$ (2002) demonstrated that evidence of a mixed phenotype suggests the transition and differentiation of cells through metaplasia. This transition from one acinus to a duct may be a result of epithelial metaplasia, which is defined as the transition from a reactive differentiated epithelium to another type of differentiated epithelium characterized by an impaired function or an alteration that is not physiologically normal in that place ${ }^{13}$. Salivary glands exhibit a variety of metaplastic changes ${ }^{13}$ and this could explain the cellular phenotypic changes resulting from aging. For Mintz and Mooradian ${ }^{17}$ (1987), the sublingual glands underwent squamous metaplasia of the ductal epithelium with age. According to Ihrler, et al. ${ }^{13}$ (2002), the majority of salivary gland metaplasias develop from basal cells of striated and excretory ducts that have an enormous capacity for pluridirectional morphogenetic differentiation. For us, our results strongly suggest metaplasia to explain this agerelated change in human salivary glands. We believe in a dynamic process that arises in the acinus and culminates in a complete transformation of a duct. Because the acinar cells originate from the primitive ductal cells, the embryological phenomenon maybe the way that the reverse process can occur.

Extrapolating this data to the clinic, we understand that the acini fail to work as secretory units and start to conduct the saliva. At this moment, they have the DLS phenotype. This could explain the decrease in the function of the saliva with age. According to Dayan, et al. ${ }^{6}$ (2000) and Vered, et al. $^{29}$ (2000), the decrease in acini volume may explain the decrease in secretion by the salivary glands with age. According to $\operatorname{Scott}^{23}$ (1977), the junction of all age-related microscopic changes may explain the decrease in glandular function. However, despite proof that acini decrease with age, certain studies support that saliva gland functions remain stable in healthy elderly patients ${ }^{17,25}$. Sorensen, et al. ${ }^{27}$ (2014) demonstrated that symptoms of oral dryness (xerostomia) are not associated with age-related degenerative changes in human labial salivary glands. The authors suggest that the autonomic innervation of labial salivary glands may play a role in the occurrence of xerostomia and that the integrity of labial salivary gland acini is related to the parenchymal autonomic innervation ${ }^{27}$. One 
hypothesis is that the secretion reserve capacity of the glands can be affected by external factors such as radiation or drug use, but not by senescence ${ }^{5}$. Because there is a difficulty in collecting saliva coming from the sublingual gland and lacked studies with direct tests of salivary flow ${ }^{18}$, we cannot firmly conclude if there is a decrease in salivary flow of the sublingual gland with age.

Although the clinical impact of the age-related changes in the salivary glands is still unclear, we think this work represents a contribution to pathologists. Because these structures are commonly found in mouth floor, pre-prosthetic surgery specimens and in neoplasias of the major and minor salivary glands, these findings may help explain the phenomenon of the appearance of multiple DLS when elaborating microscopic pathology reports.

\section{CONCLUSION}

The present study demonstrated that duct-like structures present a ductal phenotypic profile and do not present cell proliferation activity. They may represent a regressive process arising from acini or represent the result of metaplasia.

\section{ACKNOWLEDGEMENTS}

The authors would like to thank PROAP and CAPES for financial support. We would also like to thank the professors and employees of the Department of Biological Sciences (Histology) at the Bauru School of Dentistry, and Dr. Vanessa Soares Lara, Dr. Tânia Mary Cestari and Dr. Rumio Taga for their contributions to this study.

\section{CONFLICT OF INTEREST STATEMENT}

The authors declare no conflicts of interest with respect to the authorship and publication of this article.

\section{REFERENCES}

1- Araújo VC, Sousa SO, Carvalho YR, Araújo NS. Application of immunohistochemistry to the diagnosis of salivary gland tumors. Appl Immunohistochem Mol Morphol. 2000;8(3):195-202.

2- Azevedo LR, Damante JH, Lara VS, Lauris JRP. Age-relates changes in human salivary glands: a post mortem study. Arch Oral Biol. 2005;50(6):565-74.

3- Born IA, Schwechheimer K, Maier H, Otto HF. Cytokeratin expression in normal salivary glands and in cystadenolymphomas demonstrated by monoclonal antibodies against selective cytokeratin polypeptides. Virchows Arch A Pathol Anat Histopathol. 1987;411(6):583-9.

4- Brown DC, Gatter KC. Monoclonal antibody Ki-67: its use in histopathology. Histopathology. 1990;17(6):489-503.
5- Burford-Mason AP, Cummins MM, Brown DH, Mackay AJ, Dardick I. Immunohistochemical analysis of the proliferative capacity of duct and acinar cells during ligation induced atrophy and subsequent regeneration of rat parotid gland. J Oral Pathol Med. 1993;22(10):440-6.

6- Dayan D, Vered M, Paz T, Buchner A. Aging of human palatal salivary glands: a histomorphometric study. Exp Gerontol. 2000;35(1):85-93.

7- Dayan D, Vered M, Sivor S, Hiss $Y$, Buchner A. Age-related changes in proliferative markers in labial salivary glands: a study of argyrophilic nucleolar organizer regions (AgNORs) and Ki-67. Exp Gerontol. 2002;37(6):841-50.

8- De Wilde PC, Baak JP, van Houwelingen JC, Karter L, Slootweg PJ. Morphometric study of histological changes in sublabial salivary glands due to age process. J Clin Pathol. 1986;39(4):406-17.

9- Drummond JR, Chisholm DM. A qualitative and quantitative study of the ageing human labial salivary glands. Arch Oral Biol. $1984 ; 29(2): 151-5$.

10- Geiger S, Geiger B, Leitner O, Marshak J. Cytokeratin polypeptides expression in different epithelial elements of human salivary glands. Virshows Arch A Pathol Anat Histopathol. 1987;410(5):403-14.

11- Gerdes J, Becker MH, Key G, Cattoretti G. Immunohistological detection of tumor growth fraction (Ki-67 antigen) in formalin fixed and routinely tissues. J Pathol. 1992;168(1):85-6.

12- Goulart MC, Freitas-Faria P, Goulart GR, Oliveira AM, CarlosBregni R, Soares CT, et al. Pleomorphic adenoma with extensive squamous metaplasia and keratin cyst formations in minor salivary gland: a case report. J Appl Oral Sci. 2011;19(2):182-8.

13- Ihrler S, Zietz C, Sendelhofert A, Lang S, Blasenbreu-Vogt S, Löhrs U. A morphogenetic concept of salivary duct regeneration and metaplasia. Virchows Arch. 2002;440(5):519-26.

14- Iwaki Filho L, Damante JH, Consolaro A, Bonachela WC, Damante CA. Mouth floor enlargements related to the sublingual glands in edentulous or partially edentulous patients: a microscopic study. J Appl Oral Sci. 2006;14(4):264-9.

15- Maria OM, Maria SM, Redman RS, Maria AM, Saad El-Din TA, Soussa EF, et al. Effects of double ligation of Stensen's duct on the rabbit parotid gland. Biotech Histochem. 2014;89(3):181-98. 16- Marshak G, Leitner O. Cytokeratin polypeptides in normal and metaplastic human salivary gland epithelia. J Oral Pathol. $1987 ; 16(9): 442-9$.

17- Mintz GA, Mooradian AD. Age-related changes in rat sublingual salivary gland morphology. Gerodontol. 1987;6(4):137-44.

18- Moreira CR, Azevedo LR, Lauris JRP, Taga R, Damante JH. Quantitative age- related differences in human sublingual gland. Arch Oral Biol. 2006;51(11):960-6.

19- Nakazato Y, Ishida Y, Takahashi K, Suzuki K. Immunohistochemical distribution of $\mathrm{S}-100$ protein and glial fibrillary acidic protein in normal and neoplastic salivary glands. Virchows Arch A Pathol Anat Histopathol. 1985;405(3):299-310. 20- Nery LR, Moreira CR, Cestari TM, Taga R, Damante $\mathrm{JH}$. Postmortem acinar autolysis in rat sublingual gland: a morphometric study. J Appl Oral Sci. 2010;18(5):509-14.

21- Ninomiya T, Naito R, Okada Y, Kobayashi K, Mori M, Tsukitani K. Immunohistochemical localization of the alpha and beta subunits of S-100 protein in pleomorphic adenoma of the salivary glands. Virchows Arch B Cell Pathol Incl Mol Pathol. 1989;57(1):63-75. 22- Sá JC, Tolentino ES, Azevedo-Alanis LR, Iwaki-Filho L, Lara VS, Damante JH. Morphology and morphometry of the human sublingual glands in mouth floor enlargements of edentulous patients. J Appl Oral Sci. 2013;21(6):540-6.

23- Scott J. Quantitative age changes in the histological structure of human submandibular salivary glands. Arch Oral Biol. $1977 ; 22(3): 221-7$.

24- Scott J, Bodner L, Baum BJ. Assessment of age-related changes in the submandibular and sublingual salivary glands of the rat using stereological analysis. Short communication. Arch Oral Biol. 1986;31(1):69-71. 
25- Scott J, Flower EA, Burns J. A quantitative study of histological changes in the human parotid gland occurring with adult age. J Oral Pathol. 1987;16(10):505-10.

26- Soini $Y$, Puhakka A, Kahlos K, Säily M, Pääkkö $P$, Koistinen $P$, et al. Endothelial nitric oxide synthase is strongly expressed in malignant mesothelioma but does not associate with vascular density or the expression of VEGF, FLK1 or FLT1. Histopathology. 2001;39(2):179-86.

27- Sørensen CE, Larsen JO, Reibel J, Lauritzen M, Mortensen EL, Osler M, et al. Associations between xerostomia, histopathological alterations, and autonomic innervation of labial salivary glands in men in late midlife. Exp Gerontol. 2014;57:211-7.
28- Takahashi S, Shinzato K, Nakamura S, Domon T, Yamamoto T, Wakita M. The roles of apoptosis and mitosis in atrophy of the rat sublingual gland. TissueCell. 2002;34(5):297-304.

29- Vered M, Buchner A, Boldon P, Dayan D. Age-related histomorphometric changes in labial salivary glands with special reference to the acinar component. Exp Gerontol. 2000;35:107584.

30- Waterhouse JP, Chisholm DM, Winter RB, Patel M, Yale RS. Replacement of functional parenchymal cells by fat and connective tissue in human submandibular salivary glands: an age-related change. J Oral Pathol. 1973;2(1):16-27. 